\title{
Composite Material Surface Analysis based Image Texture Analysis
}

\author{
Abdul Adeem Zaily \\ Hameed \\ College of Computer Science \\ and \\ Information Technology \\ Department of Computer \\ Science \\ Anbar University/ Anbar, Iraq
}

\author{
Faik Hammad Anter \\ College of Sciences \\ Department of physics \\ Anbar University/ Anbar, Iraq
}

\author{
Muzhir Shaban Al-Ani \\ College of Computer Science \\ and \\ Information Technology \\ Department of Computer \\ Science \\ Anbar University/ Anbar, Iraq
}

\begin{abstract}
Now a days surface analysis have many application in various knowledge fields and one of the important field is material surface analysis. This approach is concentrated on the image enhancement to arise and detect small obstacles during the surface. This approach allows individual features and details of the specimen to become visible In addition many statistical measures are applied to evaluate this performance of this approach. In the obtained measures, the signal-to-noise ratio must be large enough to be interpreted in terms of the formation of a coherent image.
\end{abstract}

\section{Keywords}

Image Analysis, Surface Analysis, Intensity Analysis, Optical Analysis and Statistical Measures.

\section{INTRODUCTION}

An image lacks contrast when there are no sharp differences between black and white. Brightness refers to the overall lightness or darkness of an image. Contrast is defined as the separation between the darkest and brightest areas of the image. Increase contrast means increase the separation between dark and bright, making shadows darker and highlights brighter. Decrease contrast and you bring the shadows up and the highlights down to make them closer to one another. Adding contrast usually makes an image look more vibrant while decreasing contrast can make an image look unacceptable [1]. When imaging specimens in the optical microscope, differences in intensity and/or color create image contrast, which allows individual features and details of the specimen to become visible.

Contrast is defined as the difference in light intensity between the image and the adjacent background relative to the overall background intensity. In general, a minimum contrast value of $2 \%$ is needed by the human eye to distinguish differences between the image and its background [2].

Recently image processing tools and application become an important issue in many fields including image enhancement, restoration, reconstruction, feature Extraction, recognition and compression that affected our day life. In this work we will try to apply the powerful of image processing tools in enhancement and recognition of the compound material surface.

\section{IMAGE PROCESSING ASPECTS}

Image processing and computer vision are typical important fields of information science and technology. Several areas of mathematics have contributed to essential progress of these fields.
Image processing is a method to convert an image into digital form and perform some operations on it, in order to get an enhanced image or to extract some useful information from it. It is a type of signal dispensation in which input is image, like video frame or photograph and output may be image or characteristics associated with that image. Usually image processing system includes treating images as two dimensional signals while applying already set signal processing methods to them $[3,4,5]$.

The fundamental of texture related applications as texture analysis seeks to derive a general, efficient and compact quantitative description of textures so that various mathematical operations can be used to alter, compare and transform textures. Most available texture analysis algorithms involve extracting texture features and deriving an image coding scheme for presenting selected features. These algorithms might differ in either which texture features are extracted or how they are presented in the description. A statistical approach describes a texture via image signal statistics which reflect nondeterministic properties of spatial distribution of image signals. A spectral method extracts texture features from the spectral domain. A structural approach considers a texture as a hierarchy of spatial arrangements of well-defined texture primitives. A probability model describes the underlying stochastic process that generates textures $[6,7]$.

An image is a two dimensional (2D) function $f(x, y)$, where $x$ and $y$ are spatial coordinates and the magnitude of $f$ at any point is called the intensity of the image at that point. When $\mathrm{x}, \mathrm{y}$ and the intensity are discrete quantities we call the $\mathrm{x}, \mathrm{y}$ and the intensity are discrete quantities we call the image a digital image. The elements of a digital image are referred to as pixels. An image which has $\mathrm{M}$ rows and $\mathrm{N}$ columns is defined to be an image of size $\mathrm{M} \times \mathrm{N}$. An image is an array, or a matrix, of square pixels (picture elements) arranged in columns and rows. In a (8-bit) gray scale image each picture element has an assigned intensity that ranges from 0 to 255 . A grey scale image is what people normally call a black and white image, but the name emphasizes that such an image will also include many shades of gray [3,4]. MATLAB image processing toolbox provides a comprehensive set of reference standard algorithms, functions, and apps for image processing, analysis, visualization, and algorithm development. You can perform image analysis, image segmentation, image enhancement, noise reduction, geometric transformations, and image registration. 


\section{RELATED WORKS}

Many related works are published in this subject, and we considered the following works:

N. Lvova et. al. (2011) implemented algorithms for automatic analysis of the geometrical parameters of surface images obtained by scanning probe microscopy have been developed. The algorithms include calibration of linear displacements of the scanners using the image of the periodic linear reference samples. The algorithm based on calculation and analysis of geometrical parameters of the imprints and traces left after the surface deformation during scratch test and indentation The experimental data presented demonstrate the performance of the software modules based on the proposed algorithms [8].

Ersin Kayahan et. al. (2010), presented results from an optical technique for measuring surface roughness using image analysis of speckle pattern images. The technique coined as statistical properties of binary images (SPBI) utilizes the combine defects of speckle and scattering phenomena. The speckle patterns obtained with a $\mathrm{He}-\mathrm{Ne}$ laser were binarized and examined. The proposed method for the surface roughness combined with a non-contact optical measuring system is applied to samples from 0.5825 to $1.9 \mathrm{~mm}$ of steel (CK 45) through CNC face-milling process [9].

Xianghua Xie (2008) reviewed recent advances in surface inspection using computer vision and image processing techniques, particularly those based on texture analysis methods. This paper focused on a particular but important subset which generally treats visual surface inspection as texture analysis problems. Other topics related to visual inspection such as imaging system and data acquisition are out of the scope of this survey. This paper also compared classification approaches with novelty detection approaches at the decision making stage [10].

H. Elbehiery et. al. (2007) proposed a method to divide the ceramic tile manufacturing into color analysis, dimension verification, and surface defect detection, which is the main purpose of this work. This work based on quality control enhancement by integrating a visual control stage using image processing and morphological operation techniques before the packing operation to improve the homogeneity of batches received by final users. The automated sorting systems would bring numerous benefits to the entire sector with major economic advantages, also guarantee product quality, increase plant efficiency and reduce fixed and periodic investments [11].

Xiuwen Liu and DeLiang Wang (2003), employed a spectral histogram as a feature statistic for texture classification based on a local spatial/frequency representation. A filter selection algorithm is proposed to maximize classification performance of a given dataset. The classification experiments using natural texture images reveal that the spectral histogram representation provided a robust feature statistic for textures and generalizes well [12].

\section{COMPOSITE MATERIAL}

Epoxy Resins are thermosetting polymers that are widely used in adhesives, pains, coatings, medical implants, and electrical devices. Epoxy is also widely used as a matrix material in fibrous composites for the aerospace and wind turbine industries. Epoxies are ideal for these applications because of their high specific stiffness, high specific strength electrical installation properties, corrosion resistance, chemical compatibility with reinforcing fibers, and relative ease of manufacture.

The compound mixture of Matrix Epoxy Resin is molded by adding Alumina (Al2O3) with percentage weight of $4 \%, 6 \%$ and $8 \%$, these models are tested of $1 \mathrm{~cm}, 0.6 \mathrm{~cm}$ and $0.3 \mathrm{~cm}$ thickness. $\mathrm{Al} 2 \mathrm{O} 3$ is available from ceramaret with a purity of up to $99.9 \%$. It is a polycrystalline in material with grain size of 1 to 5 microns, with an average density of $3.95 \mathrm{~g} / \mathrm{cm} 3$ and a hardness of about 2000 Vickers.

\section{THE PROPOSED APPROACH}

The proposed image processing of surface analysis approach based on increasing features and details of the specimen to become more visible. Many steps are done to implement the procedure of construct the hybrid composite material via molding steps. Then images are taken before and after drying the specimen with the certain oven during a specific time. The main steps of the image processing system to highlight the details of the specimen in order to be ready for decision are described in figure (1). These steps are:

- Image Acquisition: including converting the optical image into digital form to be ready for processing.

- Image Prepressing: including image resizing, and converting image into gray scale image.

- Feature Extraction: including enhancing images to be ready for extracting their features.

- $\quad$ Pattern Classification: including splitting image into groups to be ready for classification.

- Identify Objects: including detect and identify even small objects in the image.

- Decision Making: including the comparison between data to reach the correct decision.

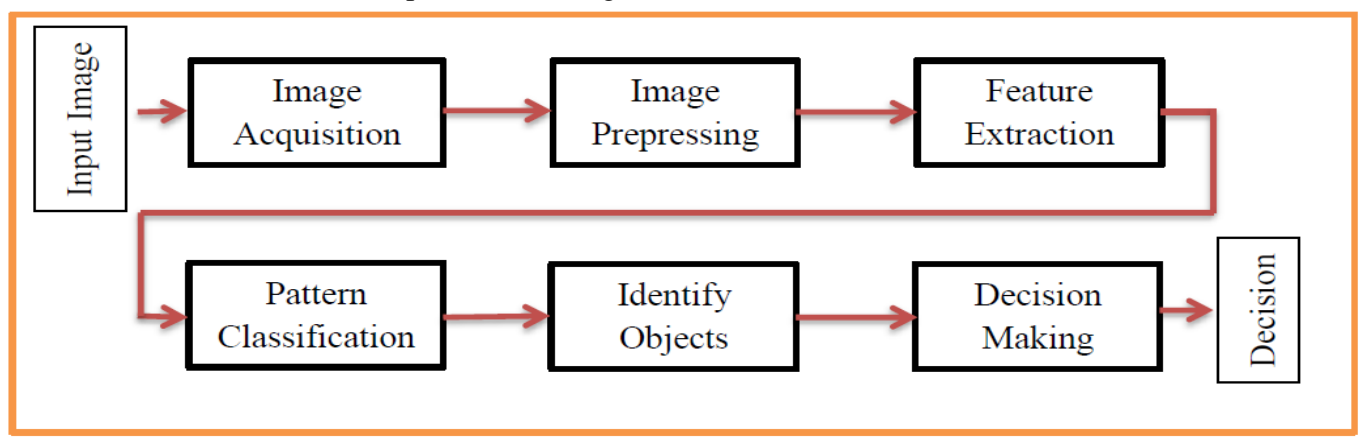

Figure (1) image processing approach 


\section{RESULTS AND ANALYSIS}

The proposed approach is applied for images before and after curing so we will try to evaluate these images in order to extract the specific features. We will demonstrate one model to explain the effect of image processing to extract its performance. Figure (2) shows $4 \%$ percentage weight, model (A) of $1.0 \mathrm{~cm}$ thick which is the original image before and after curing and their histogram in which we see that there is no difference between the two images. After we enhanced these two images via applying image processing

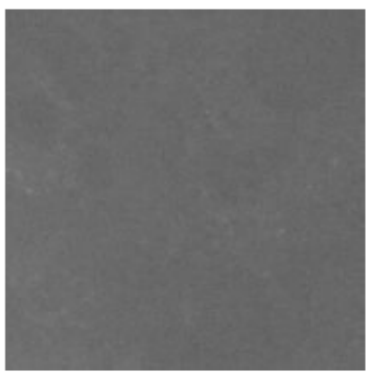

(a) image before curing

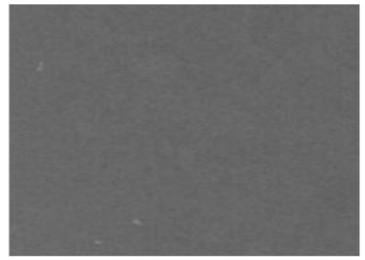

(c) image after curing enhancement, we can see that there is a bit difference between image (a) and image (b) as shown in figure (3). The difference can be seen clearly when we applied the contour processing that illuminate the details of the image as shown in figure (4). In addition figures (5) and (6) show more details about model (B) $0.6 \mathrm{~cm}$ and model (C) $0.3 \mathrm{~cm}$ thick respectively in which the curing images show more symmetrical than the other. One point can be illustrated here that model (B) may have some distortion in molding process that can be indicated in the obtained results.

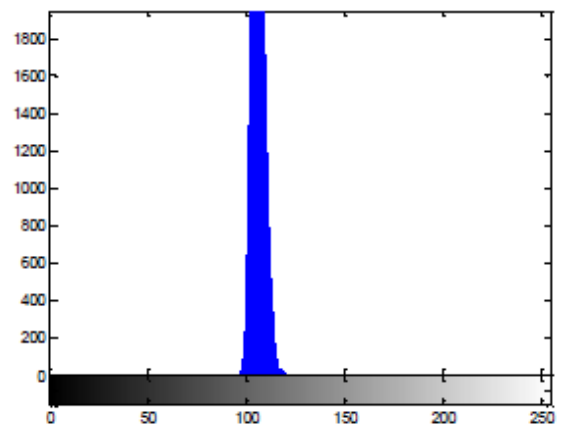

(b) histogram of the image before curing

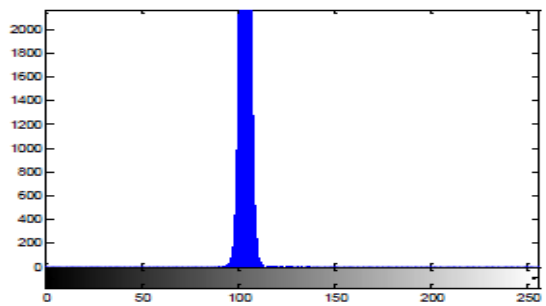

(d) histogram of the image after curing

Figure (2) original image before and after curing and their histogram

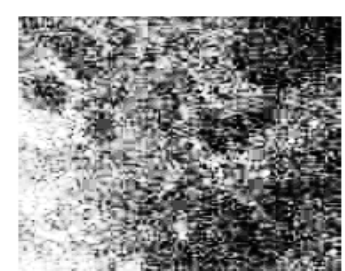

(a) enhanced image before curing

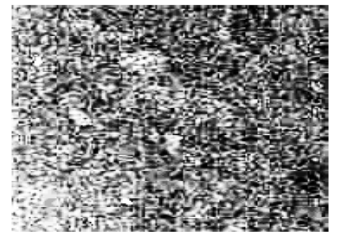

(c) enhanced image after curing

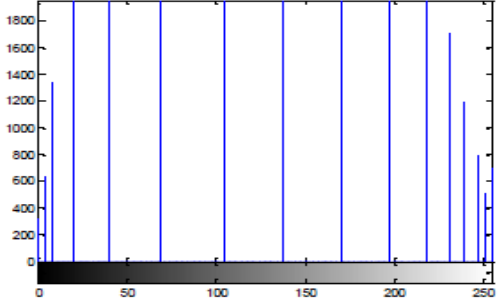

(b) histogram of the enhanced image before curing

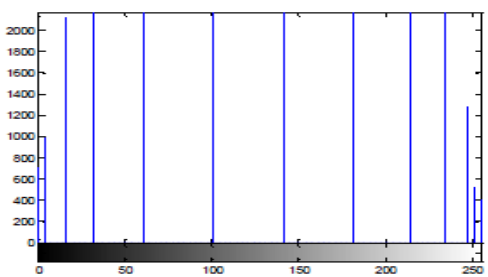

(d) histogram of the enhanced image after curing

Figure (3) enhanced image before and after curing and their histogram 


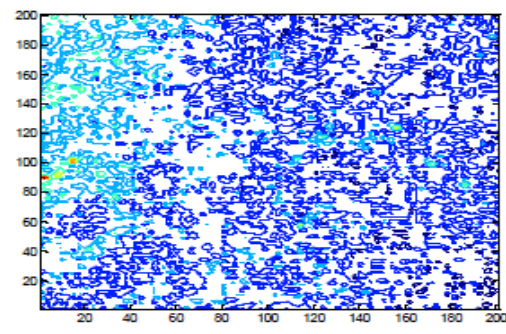

(a) contour of image before curing

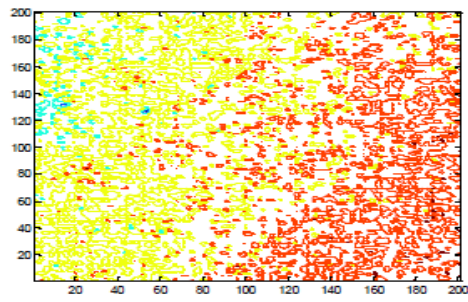

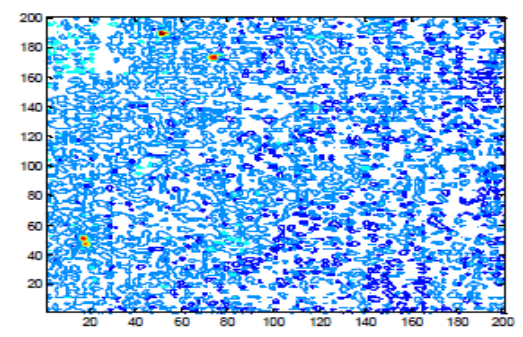

(b) contour of image after curing

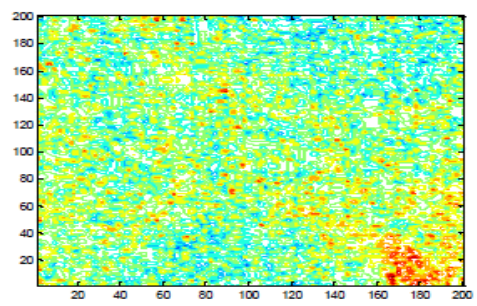

Figure (4) contour of image before and after curing $(1 \mathrm{~cm})$
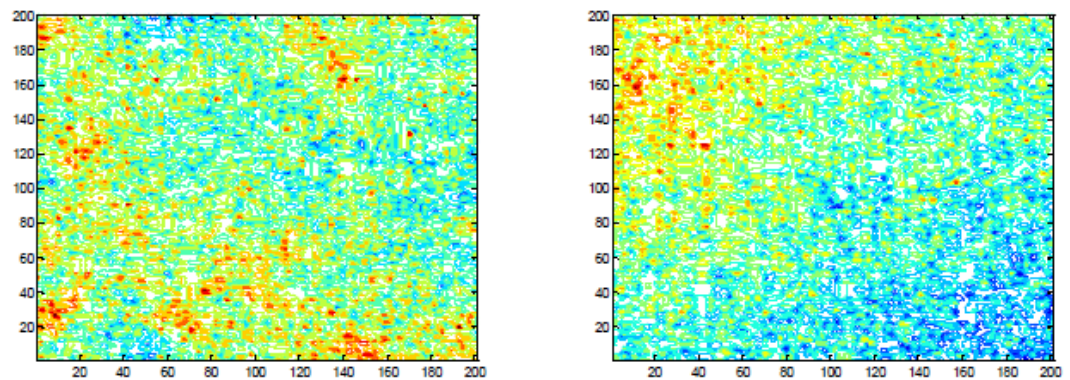

Figure (5) contour of image before and after curing $(0.6 \mathrm{~cm})$

Table (1) statistical measures of $4 \%$ Alumina percentage

\begin{tabular}{|c|c|c|c|c|c|}
\hline Model Type & PSNR $^{1}$ & MSE $^{2}$ & MAXERR $^{3}$ & L2RAT $^{{ }^{2}}$ & CORR $^{3}$ \\
\hline $\begin{array}{c}\text { Model (A) } \\
1.0 \mathrm{~cm}\end{array}$ & 35.6509 & 17.7009 & 30.0000 & 0.9573 & 0.2150 \\
\hline $\begin{array}{c}\text { Model (B) } \\
0.6 \mathrm{~cm}\end{array}$ & 36.6832 & 13.9560 & 20.0000 & 1.0274 & 0.1039 \\
\hline $\begin{array}{c}\text { Model (C) } \\
0.3 \mathrm{~cm}\end{array}$ & 24.9430 & 208.3432 & 29.0000 & 0.7995 & 0.0250 \\
\hline
\end{tabular}

Where :

PSNR1 = peak signal-to-noise ratio,

MSE2= mean square error,

MAXERR3 = maximum squared error,

L2RAT4 $=$ ratio of squared norms, and

CORR5 $=$ Correlation between images before and after curing

\section{CONCLUSIONS}

The main purpose of this work is to provide a sense of applying digital image processing in the field of compound material enhancement and detection. In this work we can denote that digital image processing becomes an efficient tool for details enhancement of material surface. Many algorithms and functions are implemented in this work to perform digital image processing issue in order to reach the main goal of the work such as feature extraction process and classification process.

Several blends of polymers that varied concentrations (4\%, $6 \%$ and $8 \%$ ) of poly were prepared to test the system. The comparison between images before and after curing shows a bit difference between images, and the curing image gives better concentration. On the other hand the thickness of the model has small effect of the surface side.

\section{REFERENCES}

[1] Maria Petrou, Pedro Garcia Sevilla (2006) "Image Processing: Dealing With Texture”,Wiley; 1st Ed., 2006.

[2] Jitendra Malik, Serge Belongie, Thomas Leung and Jianbo Shi (2001), "Contour and Texture Analysis for Image Segmentation", International Journal of Computer Vision 43(1), 7-27, 2001.

[3] Rafael C. Gonzalez, Richard E. Woods, Steven L. Eddins (2009) "Digital Image Processing Using MATLAB", 2nd Ed. Gatesmark Publishing, 2009.

[4] Gonzalez and Woods (2008) "Digital Image Processing", 3rd Ed. (DIP/3e) Prentice Hall,2008.

[5] Maria Petrou , Costas Petrou (2010), "Image Processing: The Fundamentals", Wiley; 2nd Ed., 2010.

[6] Trygve Randen and John H?akon Hus?y (1999), "Texture Segmentation Using Filters with Optimized 
Energy Separation", IEEE Transactions on Image Processing, Vol. 8, No. 4, April 1999.

[7] C. H. Chen, L. F. Pau, P. S. P. Wang (eds.) (1998, "The Handbook of Pattern Recognition and Computer Vision", 2nd Edition), pp. 207-248, World Scientific Publishing Co., 1998.

[8] N. Lvova, I. Shirokov, A. Useinov, K. Gogolinskiy (2011), "Surface Image Processing Algorithms in Scanning Probe Microscopy and Nanohardness Measurements", Proceedings of the World Congress on Engineering 2011 Vol II, WCE 2011, July 6 - 8, 2011, London, U.K.

[9] Ersin Kayahan, Hasan Oktem, Fikret Hacizade, Humbat Nasibov, Ozcan Gundogdu (2010), "Measurement of Surface Roughness of Metals Using Binary Speckle Image Analysis", Tribology International 43 (2010) 307 -311 .

[10] Xianghua Xie (2008), "A Review of Recent Advances in Surface Defect Detection Using Texture analysis Techniques", Electronic Letters on Computer Vision and Image Analysis 7(3):1-22, 2008.

[11] H. Elbehiery, A. Hefnawy, and M. Elewa (2007), "Surface Defects Detection for Ceramic Tiles Using Image Processing and Morphological Techniques", World Academy of Science, Engineering and Technology 52007

[12] Xiuwen Liu and DeLiang Wang (2003), "Texture Classification Using Spectral Histograms", IEEE
Transactions on Image Processing, Vol. 12, No. 6, June 2003.

\section{AUTHORS PROFOLE}

Muzhir Shaban Al-Ani has received Ph. D. in Computer \& Communication Engineering Technology, ETSII, Valladolid University, Spain, 1994. Assistant of Dean at Al-Anbar Technical Institute (1985). Head of Electrical Department at Al-Anbar Technical Institute, Iraq (1985-1988), Head of Computer and Software Engineering Department at AlMustansyria University, Iraq (1997-2001), Dean of Computer Science (CS) \& Information System (IS) faculty at University of Technology, Iraq (2001-2003). He joined in 15 September 2003 Electrical and Computer Engineering Department, College of Engineering, Applied Science University, Amman, Jordan, as Associated Professor. He joined in 15 September 2005 Management Information System Department, Amman Arab University, Amman, Jordan, as Associated Professor, then he joined computer science department in 15 September 2008 at the same university. He joined in August 2009 College of Computer Science, Al-Anbar University, AlAnbar, Iraq, as Professor.

Abdul Adeem Zaily Hameed obtained his M.Sc. in Physics Science from University of Anbar. He is currently instructor of Computer Science in College of Computer ,University of Anbar,IRAQ. His research interests are, Image Processing, Pattern Recognition, Quantum Physics and Statics. 